\title{
Electrochemical Selective Detection of Uric Acid Using a Copper-modified Carbon Electrode
}

\author{
Hiroaki Sakamoto, $* 1 \dagger$ Ranko Hatsuda, ${ }^{* 1}$ Kazuhiro Miyamura, ${ }^{* 2}$ Haruki ShIRaIShI, ${ }^{* 3}$ and \\ Susumu SugIYAMA $* 4$
}

*1 Research Organization of Science \& Engineering, Ritsumeikan University, 1-1-1 Noji-higashi, Kusatsu, Shiga 525-8577, Japan

*2 R\&D Center, Horiba Ltd., 2 Miyanohigashi, Kisshoin, Minami, Kyoto 601-8510, Japan

*3 Department of Biotechnology, Ritsumeikan University, 1-1-1 Noji-Higashi, Kusatsu, Shiga 525-8577, Japan

*4 Ritsumeikan-Global Innovation Research Organization, Ritsumeikan University, 1-1-1 Noji-higashi, Kusatsu, Shiga 525-8577, Japan

\begin{abstract}
The uric acid (UA) level in (human) serum is an important indicator of several diseases; however, its electrochemical detection is difficult because the oxidation potentials of UA and ascorbic acid (AA) are very close. In this study, we have developed a simple and efficient UA detection method using a copper-modified carbon electrode. The detection principle is based on the selective oxidation of $\mathrm{AA}$ by $\mathrm{Cu}(\mathrm{II})$, wherein $\mathrm{Cu}$ (II) reacts selectively with AA but not with UA. By performing this specific reaction on an electrode surface, we have successfully distinguished the oxidation potential of UA without AA interference.
\end{abstract}

(Received October 31, 2010; Accepted January 10, 2011; Published March 10, 2011)

\section{Introduction}

Uric acid (UA) level is a well known and significant indicator of certain diseases such as gout, hyperuricaemia, and Lesch-Nyhan syndrome. ${ }^{1-4}$ Many types of UA sensors have been developed and commercialized in medical diagnostics for daily health monitoring; for example, colorimetry, spectrophotometry, and chromatography have been employed as analytical methods. ${ }^{5}$ Among the other methods, electrochemical techniques typically are more selective, less expensive, less time-consuming, and can be potentially applied to real time determination in vivo.

Voltammetric UA sensing typically suffers from interference from ascorbic acid (AA) because UA has an oxidation potential close to that of AA. Hence, in the electrochemical field, researchers have developed and demonstrated many types of sensors for UA determination even when AA is present. In general, two types of approaches have been reported: surface modification and improvements in the electrode material itself. Surface modifications are based on the immobilization of enzyme uricase, ${ }^{6-8}$ or the modification of a UA sensing matrix layer. ${ }^{9}$ On the other hand, activated electrodes, ${ }^{10}$ diamonds ${ }^{11}$ and carbon nanotube electrodes ${ }^{12}$ have been employed as novel electrode materials.

In this research, we focused on the selective oxidation of AA by $\mathrm{Cu}(\mathrm{II}){ }^{13,14}$ Further, by exploiting the well-known fact that $\mathrm{Cu}(\mathrm{II})$ oxidizes AA selectively even though UA coexists in the solution, we have discovered a novel UA detection method. In our method, a copper-modified carbon electrode is first prepared by electrolysis for application as the sensor electrode.

$\dagger$ To whom correspondence should be addressed.

E-mail: sakahiro@fc.ritsumei.ac.jp
Dissolution of metallic copper on the (carbon) electrode is then performed by applying an anodic potential, and $\mathrm{Cu}(\mathrm{II})$ is locally supplied provided to the adjacent surface. The selective oxidation of $\mathrm{AA}$ is performed by the dissolved $\mathrm{Cu}(\mathrm{II})$. In this manner, UA can be detected selectively because AA becomes an electrochemically inactive molecule due to the oxidation by $\mathrm{Cu}(\mathrm{II})$. The scheme for our electrochemical-selective UA detection system is shown in Fig. 1.

We will now describe the development of the novel UA sensor and our experimental investigations into its applicability.

\section{Experimental}

\section{Reagents and materials}

UA, AA, and copper(II) chloride dihydrate were purchased from Wako Pure Chemical Industries, Ltd. (Osaka, Japan); all reagents were guaranteed pure grade.

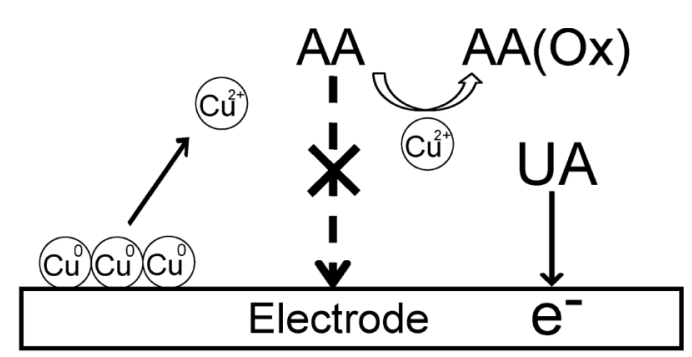

Fig. 1 Hypothetical illustration of uric acid detecting system, based on selective oxidation of ascorbic acid by $\mathrm{Cu}$ (II). 


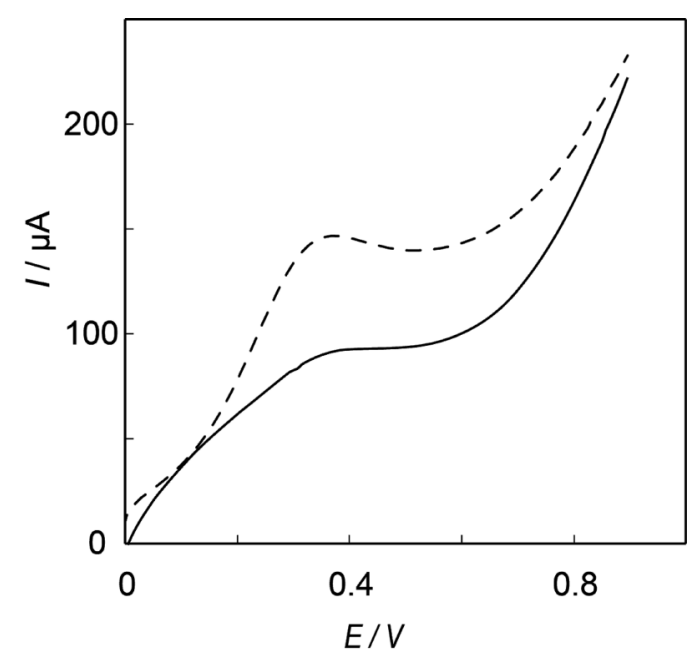

Fig. 2 Linear sweep voltammograms of AA at the bare carbon electrode (dashed line) and a copper-deposited electrode (solid line) in phosphate buffer saline at a scan rate of $50 \mathrm{mV} \mathrm{s}^{-1}$ and an AA concentration of $10 \mathrm{mg} / \mathrm{dL}$.

A Pt coil electrode was obtained from BAS Inc. (Tokyo, Japan). Carbon sheets (thickness $=100 \mu \mathrm{m}$ ) were obtained from Toyo Tanso Co., Ltd. (Osaka, Japan). The water used in the present experiment was deionized and passed through a Milli-Q water purification system from Millipore Co. (Bedford, MA).

Electrochemical fabrication of sensor electrode and electrochemical measurements

Electrochemical experiments were performed using an electrochemical analyzer (HZV-100, HOKUTODENKO, Tokyo, Japan). The three-electrode system consisted of the carbon sheet working electrode $(5 \times 5 \mathrm{~mm})$, a platinum wire counter electrode and an $\mathrm{Ag} / \mathrm{AgCl}$ reference electrode $(3 \mathrm{M}-\mathrm{NaCl})$, to which all potentials were referred. Copper chloride was dissolved in a $5-\mathrm{ml} \mathrm{KCl}$ solution to a final concentration of $1 \mathrm{mM}$. Electrochemical copper plating was controlled by applying $-300 \mathrm{mV}$ vs. $\mathrm{Ag} / \mathrm{AgCl}$ for $1 \mathrm{~min}$. Electrochemical copper dissolution from the electrode surface was controlled by applying $+100 \mathrm{mV}$ for $30 \mathrm{~s}$. For the electrochemical detection of UA, phosphate buffer saline (PBS) solution (0.1 M, pH 7.4) was used as the electrolyte solution. Electrochemical measurements of UA were conducted using linear sweep voltammetry (LSV) with a potential sweep speed of $50 \mathrm{mV} \mathrm{s}^{-1}$.

\section{Results and Discussion}

The present UA sensor is based on the selective oxidation reaction in which $\mathrm{Cu}$ (II) oxidizes AA selectively and excludes UA. In order to confirm the AA oxidation with the dissolved copper ions from the electrode surface, we performed LSV measurements in AA solution (Fig. 2). An anodic current peak was observed at $370 \mathrm{mV}$ with the bare carbon electrode (Fig. 2; dotted line). On the other hand, the anodic current peak drastically decreased with the copper-deposited electrode (Fig. 2; solid line). The data indicate that the AA molecule was oxidized from $\mathrm{Cu}(\mathrm{II})$ supplied by copper deposited on the electrode via electrolytic oxidation. Therefore, the oxidized AA becomes an electrochemically inactive molecule, and hence no

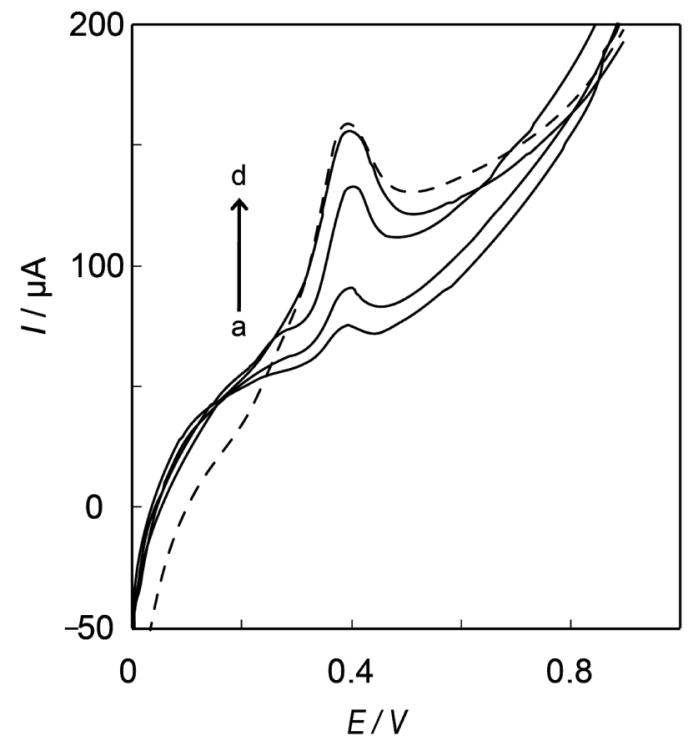

Fig. 3 LSVs obtained for the mixture containing different concentrations of UA and AA in PBS (pH 7.4) at a copper-deposited electrode. AA $(10 \mathrm{mg} / \mathrm{dL})$ and $\mathrm{UA}(\mathrm{a}-\mathrm{d}: 1.0,2.0,4.0,8.0 \mathrm{mg} / \mathrm{dL})$ (solid line); AA (5 mg/dL) and UA ( $8 \mathrm{mg} / \mathrm{dL})$ (dotted line).

AA can be detected electrochemically.

On the basis of the above results, we performed LSV measurements in a solution that included both AA and UA.

Linear sweep voltammograms (LSVs) for various concentrations of AA and UA in the mixture were plotted; the results are shown in Fig. 3. When the AA concentration was maintained constant at $10 \mathrm{mg} / \mathrm{dL}$, the UA concentration increased from 1 to $8 \mathrm{mg} / \mathrm{dL}$; further, the oxidation peak current of UA increased with the UA concentration. In contrast, when the AA concentration was decreased to $5 \mathrm{mg} / \mathrm{dL}$, the peak current of UA remained constant. Further, the calibration curve demonstrated that, although the sample solution contained AA, UA was positively proportional to its concentration. The resultant calibration curve showed excellent linearity, with $R^{2}=0.9925$. The determination limit (DL) and quantitation limit (QL) were 0.64 and $1.93 \mathrm{mg} / \mathrm{dL}$, respectively. A small shoulder peak was observed at $250 \mathrm{mV}$ vs. $\mathrm{Ag} / \mathrm{AgCl}$. The oxidation peak has been described in the literature as follows: $\mathrm{Cu}(\mathrm{II})$ is reduced by $\mathrm{AA}$ to become $\mathrm{Cu}(\mathrm{I})$, and the $\mathrm{Cu}(\mathrm{I})$ then forms a $\mathrm{Cu}(\mathrm{I})$-UA complex that precipitates onto the electrode surface. ${ }^{15,16}$ These data lead us to the conclusion that the method detects the UA concentration selectively without interference from AA.

This detection method detects UA with selectivity by using the selective oxidation of AA with $\mathrm{Cu}(\mathrm{II})$. The mechanism of selective oxidation is considered to be as follows. AA has a five-member ring structure with two adjacent hydroxyl groups. In the neutral solution, the two hydroxyl groups became strong electron donors because of the deprotonation of AA. The AA oxidation is caused by an adjacent hydroxyl group acting as an electron donor. On the other hand, UA does not have such strong electron donors. The electron donating group of AA reacts with $\mathrm{Cu}$ (II), thereby leading to selective oxidation of AA.

\section{Conclusions}

In order to detect UA selectively, we have developed a novel UA sensor using a copper-modified carbon electrode. Our sensor is 
based on a specific reaction between $\mathrm{Cu}$ (II) and AA. When AA is oxidized by copper ion, AA selectively becomes an electrochemically inactivated molecule. We experimentally demonstrated that the present sensor can perform UA detection without AA interference. Sensitivity to the UA concentration was achieved over a range of 1 to $8 \mathrm{mg} / \mathrm{dL}$, which is the reference range of UA concentrations. The present method has selectivity even though enzymes are not utilized; this implies that the present sensor has very long-term storage capability. Moreover, our novel sensor can simply and easily modify electrodes, and has practical applications as a sensor for medical diagnostics.

\section{Acknowledgements}

This work was supported by Kyoto Environmental Nanotechnology Cluster, with funding from the Ministry of Education, Culture, Sports, Science and Technology.

\section{References}

1. M. D. Harris, L. B. Siegel, and J. A. Alloway, Am. Fam. Physician, 1999, 4, 925.

2. M. Alderman and K. J. V. Aiyer, Curr. Med. Res. Opin.,
2004, 20, 369 .

3. R. J. Johnson, D.-H. Kang, D. Feig, S. Kivlighn, J. Kanellis, S. Watanabe, K. R. Tuttle, B. R. Iturbe, J. H. Acosta, and M. Mazzali, Hypertension, 2003, 41, 1183.

4. J. Fang and M. H. Alderman, JAMA, 2000, 283, 2404.

5. E. M. Strochkova, Y. I. Tur'yan, I. Kuselman, and A. Shenhar, Talanta, 1997, 44, 1923.

6. M. Shaolin, K. Jinqing, and Z. Jianbing, J. Electroanal. Chem., 1992, 334, 121.

7. S. Kuwabata, T. Nakaminami, S. Ito, and H. Yoneyama, Sens. Actuators, B, 1998, 52, 72.

8. F. Arslan, Sensors, 2008, 8, 5492.

9. L. Zheng, S. Wu, X. Lin, L. Nie, and L. Rui, Electroanalysis, 2001, 13, 1351.

10. K. Shi and K.-K. Shiu, Electroanalysis, 2001, 13, 1319.

11. E. Popa, Y. Kubota, D. A. Tryk, and A. Fujishima, Anal. Chem., 2000, 72, 1724.

12. R. Akbari, M. Noroozifar, M. K. Motlagh, and A. Taheri, Anal. Sci., 2010, 26, 425.

13. A. Hanaki, Chem. Pharm. Bull., 1969, 17, 1839.

14. K. Hayakawa, S. Minami, and S. Nakamura, Bull. Chem. Soc. Jpn., 1973, 46, 2788.

15. H. Shiraishi and R. Takahashi, Bioelectrochem. Bioenerg., 1993, 31, 203.

16. H. Shiraishi and R. Takahashi, Anal. Sci., 1994, 10, 133. 